\title{
On the statistical mechanics of an adiabatic ensemble
}

\author{
S.N.Andreev, A.A.Rukhadze ${ }^{\dagger}$ A.A.Samokhin ${ }^{\ddagger}$ \\ A.M.Prokhorov General Physics Institute of RAS, \\ 38 Vavilova Str., 119991 Moskow, Russia
}

Received December 26, 2003

Different descriptions of an adiabatic process based on statistical thermodynamics and statistical mechanics are discussed. Equality of the so-called adiabatic and isolated susceptibilities and its generalization as well as adiabatic invariants are essentially used to describe adiabatic processes in the framework of quantum and classical statistical mechanics. It is shown that distribution function in adiabatic ensemble differs from a quasi-equilibrium canonical form provided the heat capacity of the system is not constant in adiabatic process.

Key words: adiabatic process, statistical ensembles, fluctuations, linear and nonlinear response

PACS: $05.20 . \mathrm{Gg}, 05.30 . \mathrm{Ch}$

\section{Introduction}

A concept of ensemble is one of the milestones in statistical physics. For a description of equilibrium states various ensembles are used - microcanonical, canonical, grand canonical, and others, which are discussed in every fundamental course of statistical physics (see, e.g., [1-3]).

Quasiequilibrium states which are realized in adiabatic processes differ from other equilibrium states. Such adiabatic states are isolated from a heat bath but they cannot be described with a microcanonical ensemble because its initial states usually belong to canonical ensemble and are characterized by definite temperature value $T$.

It is well known that for macroscopic systems different ensembles give practically the same average values of physical (thermodynamical) quantities. For this reason, in particular, the problem of adiabatic ensemble was not investigated for a long time. One of the aspects of this problem was run into for the first time in the middle of the twentieth century when the values of the so-called adiabatic and isolated

\footnotetext{
*E-mail: andreevsn@ran.gpi.ru

${ }^{\dagger}$ E-mail: rukh@fpl.gpi.ru

${ }^{\ddagger}$ E-mail: asam@ran.gpi.ru
} 
susceptibilities were compared in the framework of linear response theory for isolated spin-systems in solids $[4,5]$.

Theory of linear response of macroscopic systems to a given external perturbation is closely connected with the fluctuation-dissipation theorem [6] which was discussed in a number of papers [7-11] with active participation of Yu.L.Klimontovich. The results of this discussion were analyzed by Yu.L.Klimontovich in [12].

Linear response of a system or its susceptibility can be expressed in terms of appropriate correlation functions [13]. This expression depends on the ensemble used in its derivation. This nontrivial dependence on adiabatic ensemble and its interesting but not well known consequences are discussed in section 2 of the present paper in the framework of nonlinear response theory for isolated paramagnetic spin system in solids. In section 3 properties of adiabatic and some other ensembles are considered on the examples of one dimensional classical systems - a constrained oscillator and a pair of identical Coulomb particles. Concluding remarks are given in final section.

\section{Nonlinear response theory and adiabatic ensemble for isolated paramagnetic spin system in solids}

Adiabatic process in macroscopic system in thermodynamical quasi-equilibrium approximation can be described with the help of canonical distribution function or density matrix of the form

$$
\rho_{\mathrm{c}}=\frac{\exp (-\alpha \hat{H})}{\operatorname{Tr} \exp (-\alpha \hat{H})}, \quad \alpha=\frac{1}{k T},
$$

where "Tr" means summation of diagonal matrix elements and Hamiltonian $\hat{H}(t)$ corresponds to its instant value which is determined by the external field magnitude $H(t)$ at the instant $t$. Temperature value $T(t)$ depends also on initial values of $H_{0}$ and $T_{0}$ and can be found from the conservation entropy condition

$$
\operatorname{Tr} \rho_{\mathrm{c}} \ln \rho_{\mathrm{c}} \equiv\left\langle\ln \rho_{\mathrm{c}}\right\rangle_{\mathrm{c}}=\text { const }
$$

or the dynamic condition

$$
\operatorname{Tr} \hat{H} \frac{\partial \rho_{\mathrm{c}}}{\partial t}=0
$$

which should be satisfied by any density matrix in accordance with quantum Liouville equation for isolated system

$$
\mathrm{i} \hbar \frac{\partial \rho}{\partial t}=[\hat{H}, \rho]
$$

In our case Hamiltonian $\hat{H}=\hat{H}_{\text {int }}-\hat{M}_{z} H(t)$ contains the term $\hat{H}_{\text {int }}$ of dipoledipole interaction between the spins and the Zeeman interaction term between the 
total magnetic moment of the system $-\hat{M}_{z} H(t)$ and the external magnetic field $H(t)=H_{0}+h(t)$. From (1) and (3) it follows

$$
\frac{\mathrm{d} \alpha}{\mathrm{d} h}=\alpha \frac{\langle\Delta \hat{H} \Delta \hat{M}\rangle_{\mathrm{c}}}{\left\langle(\Delta \hat{H})^{2}\right\rangle_{\mathrm{c}}},
$$

where $\Delta \hat{H}=\hat{H}-\langle\hat{H}\rangle_{\mathrm{c}}$ and $\Delta \hat{M}=\hat{M}-\langle\hat{M}\rangle_{\mathrm{c}}$.

In the high temperature approximation

$$
\rho_{\mathrm{c}} \simeq[\hat{I}-\alpha \hat{H}] / \operatorname{Tr} \hat{I}
$$

from (2) or (5) one obtains the following values of $\alpha,\langle\hat{M}\rangle_{\mathrm{c}}$, and adiabatic susceptibility $\chi_{\mathrm{s}}$ of the system

$$
\begin{aligned}
\alpha(t) & =\alpha_{0}\left(\frac{\operatorname{Tr} \hat{H}_{0}^{2}}{\operatorname{Tr} \hat{H}^{2}}\right)^{1 / 2}=\left(\frac{H_{i}^{2}+H_{0}^{2}}{H_{i}^{2}+H(t)^{2}}\right)^{1 / 2}, \quad \hat{H}_{0}=\hat{H}(0), \quad \alpha_{0}=\alpha(0) \\
\langle\hat{M}\rangle_{\mathrm{c}} & =\alpha(t) H(t) \frac{\operatorname{Tr} \hat{M}^{2}}{\operatorname{Tr} \hat{I}} \\
\chi_{\mathrm{s}} & =\left.\frac{\partial\langle\hat{M}\rangle_{\mathrm{c}}}{\partial h}\right|_{h=0}=\alpha_{0} \frac{\operatorname{Tr} \hat{M}^{2}}{\operatorname{Tr} \hat{I}} \frac{H_{i}^{2}}{H_{i}^{2}+H_{0}^{2}} \equiv \chi_{0} \frac{H_{i}^{2}}{H_{i}^{2}+H_{0}^{2}}
\end{aligned}
$$

where $H_{i}^{2}=\left(\operatorname{Tr} \hat{H}_{\text {int }}^{2}\right) /\left(\operatorname{Tr} \hat{M}^{2}\right)$, and $\chi_{0}$ denotes isothermal susceptibility.

In the same time from equation (4) in the first order of time-dependent perturbation theory (TPT) with respect to $h(t)$ it follows for magnetic moment $M^{(1)}$

$$
\begin{aligned}
M^{(1)} & =\frac{\alpha_{0}}{\mathrm{i} \hbar \operatorname{Tr} \hat{I}} \int_{0}^{t} h\left(t_{1}\right) \operatorname{Tr} \hat{M}(t)\left[\hat{M}\left(t_{1}\right), \hat{H}_{0}\right] \mathrm{d} t_{1}= \\
& =\frac{\alpha_{0}}{\mathrm{i} \hbar \operatorname{Tr} \hat{I}} \int_{0}^{t} h\left(t_{1}\right) \operatorname{Tr} \hat{M}_{1}(t)\left[\hat{M}_{1}\left(t_{1}\right), \hat{H}_{0}\right] \mathrm{d} t_{1}= \\
& =\alpha_{0} \frac{\operatorname{Tr} \hat{M}_{1}^{2}}{\operatorname{Tr} \hat{I}} h(t)-\int_{0}^{t} \frac{\mathrm{d} h\left(t_{1}\right)}{\mathrm{d} t_{1}} \frac{\operatorname{Tr} \hat{M}_{1} \hat{M}_{1}\left(t-t_{1}\right)}{\operatorname{Tr} \hat{I}} \mathrm{~d} t_{1}, \\
\mathrm{i} \hbar \frac{\mathrm{d} \hat{M}(t)}{\mathrm{d} t} & =\mathrm{i} \hbar \frac{\mathrm{d} \hat{M}_{1}(t)}{\mathrm{d} t}=\left[\hat{M}(t), \hat{H}_{0}\right],
\end{aligned}
$$

where $h(0)=0$. Adiabatic approximation means that the term with $\mathrm{d} h / \mathrm{d} t_{1}$ in the expression for $M^{(1)}$ can be neglected because adiabatic response should not depend on relaxation parameters. From (10) for isolated susceptibility $\chi_{\text {is }}$ one has

$$
\chi_{\mathrm{is}}=\frac{\mathrm{d} M^{(1)}}{\mathrm{d} h}=\alpha_{0} \frac{\operatorname{Tr} \hat{M}_{1}^{2}}{\operatorname{Tr} \hat{I}} .
$$

Here $\hat{M}_{1}$ denotes nondiagonal part of $\hat{M}$ in the representation where $\hat{H}_{0}$ is diagonal. The nondiagonal part $\hat{M}_{1}$ appears in equations (10), (11) because the diagonal part 
$\hat{M}_{\mathrm{d}}=\hat{M}-\hat{M}_{1}$ gives no contribution to $M^{(1)}$ and to isolated susceptibility $\chi_{\text {is }}$ in contrast to the case of isothermal susceptibility $\chi_{0}$ which contains the total operator $\hat{M}$ and differs from $\chi_{\text {is }}$ if $H_{0} \neq 0$.

Thus, two different ways of calculating the adiabatic response give two expressions (9) and (11) which are not identically equal to each other. However, for sufficiently grand ( macroscopic) systems which can sustain internal equilibrium due to internal interactions these expressions should be equal

$$
\chi_{\mathrm{s}}=\chi_{0} \frac{H_{i}^{2}}{H_{i}^{2}+H_{0}^{2}}=\chi_{\mathrm{is}}=\alpha_{0} \frac{\operatorname{Tr} \hat{M}_{1}^{2}}{\operatorname{Tr} \hat{I}} .
$$

Taking into account that $\operatorname{Tr} \hat{M}_{1}^{2}=\operatorname{Tr} \hat{M}^{2}-\operatorname{Tr} \hat{M}_{\mathrm{d}}^{2}$ one obtains from (12)

$$
\operatorname{Tr} \hat{M}_{\mathrm{d}}^{2}=\operatorname{Tr} \hat{M}^{2} \frac{H_{i}^{2}}{H_{i}^{2}+H_{0}^{2}}=\frac{\left(\operatorname{Tr} \hat{H}_{0} \hat{M}\right)^{2}}{\operatorname{Tr} \hat{H}_{0}^{2}} .
$$

The problem of equality $\chi_{\text {is }}=\chi_{\mathrm{s}}$ was discussed in many papers $[4,5,14-27]$ but it can hardly be considered as completely solved because it involves yet another complicated problem about thermodynamical equilibration in an isolated system.

It is clear that equation (12) or (13) is a special case of more general relations of this type. Consider, e.g., the case of sudden change of spin-system Hamiltonian from $\hat{H}_{0}$ to $\hat{H}$ due to abrupt change of magnetic field from initial value $H_{0}$ to the final constant value $H=H_{0}+h$, where $h$ is not necessarily small as compared with $H_{0}$. From the energy conservation relation $\operatorname{Tr} \hat{H} \rho_{\infty}=\operatorname{Tr} \hat{H} \rho_{0}, \rho_{0}=\left[\hat{I}-\alpha_{0} \hat{H}_{0}\right] / \operatorname{Tr} \hat{I}$ and supposition concerning equilibrium form of the new density matrix $\rho_{\infty}$ at sufficiently long time it follows

$$
\begin{aligned}
\rho_{\infty} & =\frac{[\hat{I}-\alpha \hat{H}]}{\operatorname{Tr} \hat{I}}, \quad \alpha=\alpha_{0} \frac{\operatorname{Tr} \hat{H}_{0} \hat{H}}{\operatorname{Tr} \hat{H}^{2}}, \\
\operatorname{Tr} \hat{M} \rho_{\infty} & =\chi_{0}\left(H_{0}+h\right) \frac{H_{i}^{2}+H_{0}\left(H_{0}+h\right)}{H_{i}^{2}+\left(H_{0}+h\right)^{2}} .
\end{aligned}
$$

On the other hand, taking into account equation (4) with $\hat{H}=$ const, one can express the new equilibrium density matrix in the form

$$
\rho_{\infty} \sim \frac{\left[\hat{I}-\alpha_{0}\left(\hat{H}_{0}\right)_{\mathrm{d}}\right]}{\operatorname{Tr} \hat{I}}
$$

where $\left(\hat{H}_{0}\right)_{\mathrm{d}}$ means diagonal part of $\hat{H}_{0}$ in the representation which diagonalizes $\hat{H}$. Such a form of $\rho_{\infty}$ means that nondiagonal with respect to $\hat{H}$ part of $\rho_{\infty}$ gives no contribution to mean values of physical quantities after retaining a new equilibrium state of the system.

From the equality of the magnetic moment mean values obtained with the help of (14) and (15) one has

$$
\operatorname{Tr} \hat{M}_{\mathrm{d}}\left(\hat{H}_{0}\right)_{\mathrm{d}}=\operatorname{Tr} \hat{M} \hat{H} \frac{\operatorname{Tr} \hat{H} \hat{H}_{0}}{\operatorname{Tr} \hat{H}^{2}} .
$$


This formula is of the same type as equation (13). However, in equation (16) diagonal parts of operators correspond to the representation where $\hat{H}$ is diagonal.

Making use of the relations similar to (13) and (16) for factorization of traces of diagonal part operator products in the higher order terms of TPT and neglecting the terms irrelevant in an adiabatic approximation, one can obtain expression (8) straightforwardly from a TPT-series for equation (4) without resort to quasiequilibrium canonical form of $\rho[28]$.

If $H_{0}=0$ and $h(0)=0$ then for $n$-th order term of TPT series $\operatorname{Tr} \hat{M} \rho(t)=$ $\sum M^{(n)}$ one has

$$
\begin{aligned}
& M^{(n)}=\operatorname{Tr} \hat{M} \rho^{(n)}= \\
& \quad=\frac{\alpha_{0}(-1)^{n}}{\operatorname{Tr} \hat{I}} \int_{0}^{t} \mathrm{~d} t_{1} \ldots \int_{0}^{t_{n-1}} \mathrm{~d} t_{n} h\left(t_{1}\right) \ldots h\left(t_{n-1}\right) \frac{\mathrm{d} h\left(t_{n}\right)}{\mathrm{d} t_{n}} \cdot \operatorname{Tr}\left\{\hat{F}^{(n-1)}\left(t, t_{1} \ldots t_{n}\right) \hat{M}\left(t_{n}\right)\right\}, \\
& \hat{F}^{(n-1)}=\left[\ldots\left[\hat{M}(t), \hat{M}\left(t_{1}\right)\right] \ldots \hat{M}\left(t_{n-1}\right)\right](\mathrm{i} \hbar)^{1-n}, \quad \hat{F}^{(0)}(t)=\hat{M}(t) .
\end{aligned}
$$

In this case operator $\hat{M}$ contains only nondiagonal part while this is not so for higher order products of $\hat{M}$.

Taking this fact into account one obtains

$$
\mathrm{i} \hbar \operatorname{Tr} \hat{F}^{(n-1)} \hat{M}\left(t_{n}\right)=\operatorname{Tr}\left\{\hat{F}_{\mathrm{d}}^{(n-2)}\left[\hat{M}\left(t_{n-1}\right), \hat{M}\left(t_{n}\right)\right]_{\mathrm{d}}\right\}+\operatorname{Tr}\left\{\hat{F}_{1}^{(n-2)}\left[\hat{M}\left(t_{n-1}\right), \hat{M}\left(t_{n}\right)\right]_{1}\right\} .
$$

The second term on the right hand side of (18) contains at least the third-order correlation function and for this reason it can be neglected in adiabatic approximation. With the help of the relation

$$
\operatorname{Tr}\left\{\hat{F}_{\mathrm{d}}^{(n-2)}\left[\hat{M}\left(t_{n-1}\right), \hat{M}\left(t_{n}\right)\right]_{\mathrm{d}}\right\}=\operatorname{Tr}\left\{\hat{F}^{(n-2)} \hat{H}_{0}\right\} \cdot \operatorname{Tr}\left\{\left[\hat{M}\left(t_{n-1}\right), \hat{M}\left(t_{n}\right)\right] \hat{H}_{0}\right\} / \operatorname{Tr} \hat{H}_{0}^{2},
$$

from (18) one has

$$
\begin{aligned}
\operatorname{Tr} \hat{F}^{(n-1)} \hat{M}\left(t_{n}\right) & \approx \frac{1}{H_{i}^{2}} \operatorname{Tr}\left\{\hat{F}^{(n-3)} \frac{d \hat{M}\left(t_{n-2}\right)}{d t_{n-2}}\right\} \frac{\mathrm{d}}{\mathrm{d} t_{n}} \varphi\left(t_{n}-t_{n-1}\right), \\
\varphi\left(t_{n}-t_{n-1}\right) & =\frac{\operatorname{Tr} \hat{M}\left(t_{n}\right) \hat{M}\left(t_{n-1}\right)}{\operatorname{Tr} \hat{M}^{2}}
\end{aligned}
$$

and after partial integration this gives for $M^{(n)}$

$$
M^{(n)} \approx \frac{\alpha_{0}(-1)^{n}}{\operatorname{Tr} \hat{I}} \frac{3}{2 H_{i}^{2}} \int_{0}^{t} \mathrm{~d} t_{1} \ldots \int_{0}^{t_{n-3}} \mathrm{~d} t_{n-2} h\left(t_{1}\right) \ldots h^{2}\left(t_{n-2}\right) \frac{\mathrm{d} h\left(t_{n-2}\right)}{t_{n-2}} \operatorname{Tr} \hat{F}^{(n-3)} \hat{M}\left(t_{n-2}\right) .
$$

Here the term containing the expression

$$
\left.\varphi\left(t_{n-1}\right) \frac{\mathrm{d} h}{\mathrm{~d} t}\right|_{0}+\int_{0}^{t_{n-1}} \mathrm{~d} t_{n} \frac{\mathrm{d}^{2} h\left(t_{n}\right)}{\mathrm{d} t_{n}^{2}} \varphi\left(t_{n}-t_{n-1}\right)
$$

is omitted because it also gives no contribution to $M^{(n)}$ in adiabatic approximation. 
Continuing this process, one has finally for $M_{\mathrm{ad}}^{(n)}$ the following expression

$$
\begin{gathered}
M_{\mathrm{ad}}^{(n)}=\quad \chi_{0} h(t)(-1)^{k+1} \frac{1 \cdot 3 \cdot 5 \ldots(2 k+1)}{2^{k+1}(k+1) !}\left(\frac{h^{2}(t)}{H_{i}^{2}}\right)^{k+1}, \\
n=2 k+3, \quad k=0,1,2 \ldots,
\end{gathered}
$$

which corresponds to $(8)$ at $H_{0}=0$.

If no adiabatic approximation is used, then with the help of the natural generalization of the above considered procedure one can obtain an integro-differential equation for nonequilibrium non-linear response of the spin system, which contains correlation functions of all orders [29]. At $H_{0}=0$ and $h(0)=0$ this equation is as follows:

$$
\begin{aligned}
H_{i}^{2} M(t) & =-\sum_{n=1,3 \ldots}^{\infty} \int_{0}^{t} \mathrm{~d} t_{1} h\left(t_{1}\right) \ldots \int_{0}^{t_{n-1}} \mathrm{~d} t_{n} h\left(t_{n}\right) \frac{\mathrm{d}}{\mathrm{d} t_{n}} G_{n}\left(t, \ldots, t_{n}\right) E\left(t_{n}\right), \\
G_{n}\left(t, t_{1}, \ldots t_{n}\right) & =\operatorname{Tr}\left[\ldots\left[\left[\hat{M}(t), \hat{M}\left(t_{1}\right)\right]_{1}, \hat{M}\left(t_{2}\right)\right]_{1} \ldots\right]_{1} \frac{\hat{M}\left(t_{n}\right)}{\operatorname{Tr} \hat{M}^{2}}(\mathrm{i} \hbar)^{(n-1)} \\
G_{1}\left(t, t_{1}\right) & =\varphi\left(t-t_{1}\right)
\end{aligned}
$$

where

$$
E=E_{0}+\int_{0}^{t} \mathrm{~d} t_{1} h\left(t_{1}\right) \frac{\mathrm{d} M\left(t_{1}\right)}{\mathrm{d} t_{1}}, \quad E_{0}=\operatorname{Tr} \hat{H}_{0} \rho(0)=-\chi_{0} H_{i}^{2}, \quad E=\operatorname{Tr} \hat{H}_{0} \rho(t)
$$

In adiabatic approximation from (24) it follows

$$
H_{i}^{2} M=-h E
$$

and with the help of (25) this gives for $E$ the equation $H^{2} \mathrm{~d} E / \mathrm{d} t=-h \mathrm{~d}(h E) / \mathrm{d} t$ which has a solution

$$
E=E_{0}\left(\frac{H_{i}^{2}}{H_{i}^{2}+h^{2}}\right)^{1 / 2}
$$

From (26) and (27) there follows the same expression for $M$ as in equation (8) at $H_{0}=0$.

In the case of sudden change of magnetic field from zero value to $h=$ const at $t>0$ equation (24) at sufficiently long times also reduces to equation (26) which in this case gives

$$
H_{i}^{2} M=-h E=-h\left(E_{0}+h M\right), \quad M=\chi_{0} h \frac{H_{i}^{2}}{H_{i}^{2}+h^{2}} .
$$

This expression for $M$ coincides with expression (14) at $H_{0}=0$. The case $H_{0} \neq 0$ was considered in [30].

It is worthwhile noting that equation (24) reduces to (26) both in adiabatic and sudden approximations in the long-time limit because only the term with the lowest 
order correlation functions $\varphi\left(t-t_{1}\right)$ at $t=t_{1}$ contributes to equilibrium or quasiequilibrium values of $M$ and $E$. This is in accordance with the known fact that the properties of equilibrium states should not depend on relaxation parameters $[7,10,12]$.

In contrast to the mean values of energy and magnetic moment its fluctuations depend on the ensemble used, i.e., on the form of the density matrix. In the canonical ensemble, energy fluctuations are determined as follows

$$
\left\langle(\Delta \hat{H})^{2}\right\rangle_{\mathrm{c}}=c \Theta^{2}, \quad C=\frac{\mathrm{d}\langle\hat{H}\rangle_{\mathrm{c}}}{\mathrm{d} \Theta}=\alpha^{2}\left\langle(\Delta \hat{H})^{2}\right\rangle_{\mathrm{c}}, \quad \Theta=\frac{1}{\alpha},
$$

where $C$ denotes the system heat capacity. However, quasi-equilibrium canonical density matrix $\rho_{\mathrm{c}}$ in general case is not a solution to equation (4) in the adiabatic approximation [31]. From supposition that $\rho_{\mathrm{c}}$ is a solution to (4) in the adiabatic process it follows that $C$ does not change in this process

$$
\frac{\mathrm{d} C}{\mathrm{~d} h}=-2 \alpha \frac{\mathrm{d}\langle\hat{H}\rangle_{\mathrm{c}}}{\mathrm{d} \alpha} \frac{\mathrm{d} \alpha}{\mathrm{d} h}+2 \alpha^{2} \frac{\mathrm{d}\langle\hat{M}\rangle_{\mathrm{c}}}{\mathrm{d} \alpha}+\alpha^{2} \operatorname{Tr} \hat{H}^{2} \frac{\mathrm{d} \rho}{\mathrm{d} h}-2 \alpha^{2}\langle\hat{H}\rangle_{\mathrm{c}} \operatorname{Tr} \hat{H} \frac{\mathrm{d} \rho}{\mathrm{d} h}=0 .
$$

The first two terms on the right hand side of (30) cancel each other in accordance with (5) while the last two terms are zero, if $\rho_{\mathrm{c}}$ is a solution to equation (4).

The condition $C=$ const in an adiabatic process is met, e.g., for ideal gases or for thermal radiation photons in perfectly reflecting cavity with slowly changing volume. For the considered spin-system in the high temperature approximation the value of $C$ is also constant in adiabatic process as it is seen from (29) and (7). In a more general case the condition $C=$ const is not met so that the density matrix (1) and equation (29) do not give a correct description of fluctuations in an adiabatic process or in an adiabatic ensemble.

For this reason it useful to consider which expression for the energy fluctuations can be obtained at less restrictive suppositions, not involving an explicit form of the density matrix in adiabatic process. From equation (4) for energy fluctuations evolution it follows

$$
\frac{\mathrm{d}}{\mathrm{d} h}\left\langle\Delta \hat{H}^{2}\right\rangle=-2\langle\Delta \hat{M} \Delta \hat{H}\rangle,
$$

where the brackets $\langle\ldots\rangle$ mean the averaging with the help of an exact density matrix. If one supposes the equality for ratios of the same fluctuations in quasiequilibrium canonical and adiabatic ensembles [32]

$$
\frac{\langle\Delta \hat{M} \Delta \hat{H}\rangle}{\left\langle\Delta \hat{H}^{2}\right\rangle}=\frac{\langle\Delta \hat{M} \Delta \hat{H}\rangle_{\mathrm{c}}}{\left\langle\Delta \hat{H}^{2}\right\rangle_{\mathrm{c}}}
$$

then from (5), (31) and (32) one obtains the equation

$$
\frac{\mathrm{d}}{\mathrm{d} h}\left\langle\Delta \hat{H}^{2}\right\rangle=-\frac{2}{\alpha} \frac{\mathrm{d} \alpha}{\mathrm{d} h}\left\langle\Delta \hat{H}^{2}\right\rangle,
$$

the solution to which can be expressed as follows

$$
\frac{\left\langle\Delta \hat{H}^{2}\right\rangle}{\left\langle\Delta \hat{H}_{0}^{2}\right\rangle_{0}}=\left(\frac{\Theta}{\Theta_{0}}\right)^{2}=\frac{C_{0}}{C} \frac{\left\langle\Delta \hat{H}^{2}\right\rangle_{\mathrm{c}}}{\left\langle\Delta \hat{H}_{0}^{2}\right\rangle_{0, c}}, \quad\left\langle\Delta \hat{H}^{2}\right\rangle=\frac{C_{0}}{C}\left\langle\Delta \hat{H}^{2}\right\rangle_{\mathrm{c}},
$$


where $\langle\ldots\rangle_{0}$ means the averaging over the initial density matrix $\rho_{0}=\rho_{\mathrm{c}}(0)$.

Formula (34) is a generalization of canonical expression (29) to the case, where the system heat capacity is not constant in an adiabatic process. The formula (34) was obtained at the third order approximation with respect to the inverse temperature in a different way [33] with the help of relations like (13) and (16) which are a generalized form of the relation $\chi_{\mathrm{s}}=\chi_{\text {is }}$.

It is interesting to note that in the high temperature limit, when $\left\langle\Delta \hat{H}^{2}\right\rangle=$ $\operatorname{Tr} \hat{H}^{2} / \operatorname{Tr} \hat{I}$ is formally independent of temperature and the ensemble used, the expression (34) coincides with (7). The formula (34) was obtained also in [34] without references to preceding papers.

The problem of statistical description of isolated system dynamical evolution is a rather complicated one in the case of macroscopic (many particle) systems. For this reason it is useful to consider an adiabatic ensemble behaviour of simple few particle systems and compare it with other ensembles. It should be mentioned that various aspects of this problem were considered in many papers (see e.g. [35-37] and references therein).

\section{Adiabatic and other ensembles of simple dynamical systems}

We consider here small systems composed of a one-dimensional oscillator and one-dimensional symmetric Coulomb pair, whose motion is limited to absolutely elastic walls on an interval $[-L, L]$.

From the general expression for an adiabatic invariant [38]

$$
G(E, L)=2 \int_{x_{\min }}^{x_{\max }} p \cdot \mathrm{d} x=2 \sqrt{2 m} \int_{x_{\min }}^{x_{\max }} \sqrt{E-U(x)} \mathrm{d} x,
$$

( $E$ is total energy of a particle, $m$ is its mass, $p$ is its momentum, $\left[x_{\min }, x_{\max }\right]$ is the region of variation of the coordinate $x$ admissible for the particle motion) for a one-dimensional harmonic oscillator with Hamiltonial $H(p, r)=p^{2} / 2 m+m \omega^{2} x^{2} / 2$, limited by adiabatically moving apart absolutely elastic walls on the interval $[-L, L]$ there follows the expression

$$
G=G_{1}(y, L)=2 m \omega L^{2}\left(\sqrt{y-1}+y \cdot \operatorname{Arcsin}\left(\frac{1}{\sqrt{y}}\right)\right),
$$

where $y=E / U(L)=v^{2} / \omega^{2} L^{2}+1 \geqslant 1$ is the mechanical parameter of system perfection, $U(L)$ is the potential energy of the particle at the point $L$, and $v$ is the particle velocity close to the wall. Note, that in the considered adiabatic process, the oscillator trajectories naturally fall into three groups depending on the initial energy $E_{0}$. For $E_{0} \leqslant U\left(L_{0}\right)=m \omega^{2} L_{0}^{2} / 2$ the oscillator do not interact with the wall even in the initial position. If the initial energy $E_{0}$ is large enough, $E_{0}>E_{2}>E_{1}$ the interaction with the wall will persist in the final state as well; otherwise the oscillator 
will cease the interaction with the wall at the position $L^{\star}$ before the wall reaches the final position $L$. If $E_{0}=E_{2}$, then $L^{\star}=L$. For $E_{0}>E_{2}$ the value of $y$ can be found from the relation $G_{1}(y, L)=G_{1}\left(y_{0}, L_{0}\right)$. If $U\left(L_{0}\right)<E_{0}<E_{2}$, the $y$ value becomes unity at the point $L^{\star}<L$ determined from the condition $G_{1}\left(1, L^{\star}\right)=G_{1}\left(y_{0}, L_{0}\right)$. With a further increase of $L$ the $y$ value for a given trajectory is assumed to be equal to unity and the total energy conserves the value $E=U\left(L^{\star}\right)=m \omega L^{\star 2} / 2$ it reached at the wall position $L^{\star}$.

The adiabatic invariant of the motion of a symmetric Coulomb pair has the form

$$
G=G_{2}(y, L)=\sqrt{2 \alpha m} \frac{\sqrt{L}}{\sqrt{y}}(2 \sqrt{y(y-1)}-\operatorname{Arcch}(2 y-1)),
$$

where $y=E / U(L)=E L / \alpha$ is the mechanical parameter of system perfection and $U(L)$ is the potential energy of the particle at the point $L$. It is taken into account here that the motion of a one-dimensional Coulomb pair between elastically reflecting walls on the interval $[-L, L]$ is equivalent to the motion, bounded by the wall on the interval $[0, L]$, of a single charged particle with Hamiltonian $H(p, x)=$ $p^{2} / 2 m+\alpha / x$ in the Coulomb repulsion field with a potential center at the origin of coordinates.

In the limit $y \rightarrow 1$ the adiabatic invariant of the bounded oscillator (36) tends to well-known expression $E / \omega=$ const for the adiabatic invariant of a free oscillator with a slowly varying frequency. In the case of a symmetric Coulomb pair, in the limit $y \rightarrow 1$ expression (37) reduces to the form $\sqrt{L}(y-1)^{3 / 2}=$ const or $v L^{3 / 2}=$ const, i.e., the total energy of a Coulomb pair in an adiabatic process decreases continuously with increasing $L$, as distinct from a bounded oscillator which at a certain value $L^{\star}$ stops interacting with the wall, after which its energy $E=U\left(L^{\star}\right)$ remains unchanged.

In the inverse limit $y \rightarrow \infty$ expressions (36) and (37) tend to the expression for the adiabatic invariant of a free particle $G=4 L \sqrt{2 m E}=$ const (or $v L=$ const) whose motion is bounded by absolutely elastic walls on an interval $[-L, L]$. Note that such a simple mechanical system makes it possible to derive some relations of thermodynamics of a monoatomic ideal gas because the ideal gas model does not involve the interaction of particles among themselves, and the interaction with the walls is decisive.

Representing the temperature $\Theta$ as a double kinetic energy of the particle, which corresponds to each translational degree of freedom, expressing the mean pressure $P=2 m v / 2 S \tau$ in terms of the momentum $m v$ transferred by the particle to the wall during collision for the period $T=2 L / v$ per unit area $S=L^{k-1}(k=1,2,3$ for one-, two-, and three-dimensional cases), we obtain the equation of ideal gas state, $P V=\Theta\left(V=L^{k}\right.$ is the system volume). Then substituting the relation $v L=$ const into the formula for $P$, we arrive at the equation of the adiabatic process for this system:

$$
P V^{\gamma}=\text { const }, \quad \gamma=\frac{(k+2)}{k}, \quad k=1,2,3,
$$

in which the adiabatic index $\gamma$ is equal to 3,2 , and $5 / 3$ in respectively one-, two-, and three-dimensional cases, which corresponds to the indices known from thermodynamics of ideal gases. The mechanical interpretation of an adiabatic process for 
an ideal gas is mentioned, for example, in [39,40] for $k<3$, but is usually never considered in the presentation of thermodynamics in the courses of general and theoretical physics.

Before proceeding to a consideration of an adiabatic process, we shall compare equilibrium canonical and microcanonical ensembles for bounded oscillator and symmetric Coulomb pair. For these small systems the distinction between different ensembles manifests itself not only for fluctuations, but also for average values.

The distribution functions of a canonical and microcanonical ensembles are as follows, correspondingly:

$$
\begin{aligned}
\rho_{\mathrm{c}}(p, x) & =A \cdot \exp (-H(p, x) / \Theta), \\
\rho_{\mathrm{m}}(p, x) & =B \cdot \delta(E-H(p, x)),
\end{aligned}
$$

where the temperature $\Theta$ and the total energy $E$ are parameters of corresponding distributions. The normalization constants

$$
A=\frac{1}{\iint \exp (-H(p, x) / \Theta) \mathrm{d} p \mathrm{~d} x}
$$

and $B=1 / T(E, L)$ ( where $T(E, L)$ is period of the system oscillations) in (39) and (40) are determined from the condition of equality to unity of the integral of the distribution function over the entire range of variation of the momentum and the coordinate.

The average kinetic energy in a canonical ensemble for a given systems has its usual form $\langle T\rangle_{\mathrm{c}}=\Theta / 2$. The average value of the total energy for the bounded oscillator is described by the expression

$$
\langle E\rangle_{\mathrm{c}}=\Theta(1-h(\mu)), \quad h^{-1}(\mu)=\mathrm{e}^{1 / \mu} \sqrt{\pi \mu} \operatorname{Erf}\left(\frac{1}{\sqrt{\mu}}\right),
$$

where

$$
\mu=\frac{2 \Theta}{m \omega^{2} L^{2}}, \quad \operatorname{Erf}(x)=\frac{2}{\sqrt{\pi}} \int_{0}^{x} \exp \left(-t^{2}\right) \mathrm{d} t .
$$

The average value of the total energy for the symmetric Coulomb pair is described by the formula

$$
\langle E\rangle_{\mathrm{c}}=\frac{\Theta}{2}\left(\frac{2 \mu \mathrm{e}^{-1 / \mu}}{W(\mu)}-1\right), \quad W(\mu)=\int_{0}^{\mu} \mathrm{e}^{-1 / t} \mathrm{~d} t,
$$

where $\mu=L \Theta / \alpha$.

In the high temperature limit, where $\mu \gg 1$, expressions (41) and (42) tend to the average energy $\langle E\rangle_{\mathrm{c}}=\Theta / 2$ for a free particle and the heat capacity $C_{v}=$ $\partial\langle E\rangle_{\mathrm{c}} / \partial \Theta=1 / 2$. In the low temperature limit as $\mu \rightarrow 0$, the expression (41) tends to the average total energy of the ensemble of the free oscillators $\langle E\rangle_{\mathrm{c}}=\Theta$, and 
from the expression (42) for the symmetric Coulomb pair using the L'Hopitale rule we find $\langle E\rangle_{\mathrm{c}}=\alpha / L=U(L)$.

In the microcanonical ensemble the kinetic energy fluctuates instead of the full energy. The average kinetic energy $\langle T\rangle_{\mathrm{m}}$ with respect to the microcanonical distribution for a bounded oscillator is given by the formula

$$
\langle T\rangle_{\mathrm{m}}=\frac{E}{2}\left(1+\frac{\sqrt{y-1}}{y \cdot \arcsin (1 / \sqrt{y})}\right),
$$

where $y=E / U(L) \geqslant 1$ if $E>m \omega L^{2} / 2$ and $\langle T\rangle_{\mathrm{m}}=E / 2$, if $E \leq m \omega L^{2} / 2$. In the case of symmetric Coulomb pair the expression for the microcanonical average kinetic energy has the form

$$
\langle T\rangle_{\mathrm{m}}=E \frac{\sqrt{y(y-1)}-\ln (\sqrt{y}+\sqrt{y-1})}{\sqrt{y(y-1)}+\ln (\sqrt{y}+\sqrt{y-1})},
$$

where $y=L / 2 a=L E / \alpha$.

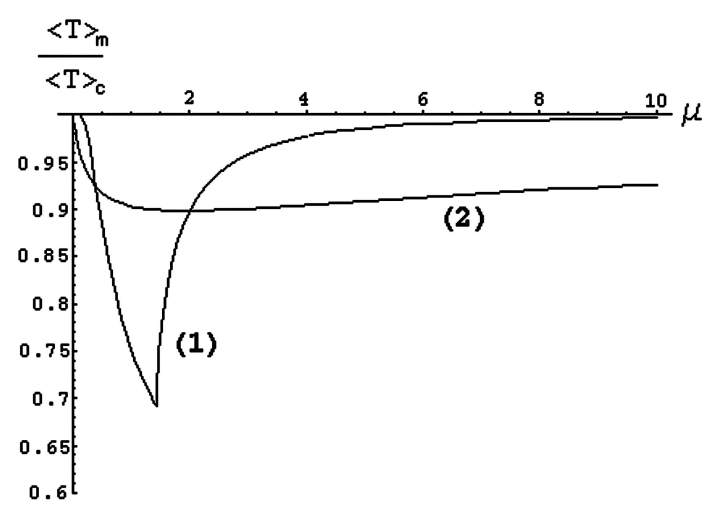

Figure 1. Ratio of average kinetic energies in a canonical and microcanonical ensembles for equal average total energies $\langle E\rangle_{\mathrm{c}}=\langle E\rangle_{\mathrm{m}}$ for bounded oscillator (curve 1) and symmetric Coulomb pair (curve 2) versus the parameter $\mu=\Theta / U(L)$.

For equal $E$ and $\langle E\rangle_{\mathrm{c}}$, in a canonical and a microcanonical ensembles the average kinetic energies $\langle T\rangle_{\mathrm{c}}$ and $\langle T\rangle_{\mathrm{m}}$ for bounded oscillators and symmetric Coulomb pairs depending on $\mu$ show (see figure 1) a substantial distinction in their behavior. In the case of the bounded oscillator (figure 1, curve 1) this distinction reaches its maximum $\langle T\rangle_{\mathrm{m}} /\langle T\rangle_{\mathrm{c}}=0.69$ at the point $\mu=1.44$, where the fluctuation distinction in canonical and microcanonical ensembles turn out to be the most considerable ( at this point "microcanonical" oscillator ceases the interaction with the walls, whereas the part of "canonical" oscillators continue to interact with the walls). In the case of symmetric Coulomb pair (figure 1, curve 2) the distinction reaches its maximum $\langle T\rangle_{\mathrm{m}} /\langle T\rangle_{\mathrm{c}}=0.9$ at the point $\mu=1.91$. In the high temperature $(\mu \gg 1)$ and low temperature $(\mu \ll 1)$ limits for both systems microcanonical and canonical average kinetic energies coincide, in spite of different forms of the distribution functions. 
In considering an adiabatic process, which is the limiting case of nonequilibrium processes, to find the distribution function one should analyze the dynamic evolution of the systems of the ensemble on the phase plane.

If the Hamiltonian of the system is independent of time, the canonical and microcanonical distribution functions are equilibrium, i.e. invariable under the dynamic evolution of the systems of the ensemble. This is explained by the fact that for fixed energy the microcanonical distribution function is uniform with respect to all the other variables, which means that it is uniform in time (time-independent). The systems under consideration are obviously ergodic, i.e., the time average is coincident with the microcanonical ensemble average. The canonical ensemble is a family of microcanonical ensembles distributed over the energy axis with a density $\sim \exp (-E / \Theta)$. The canonical distribution function is therefore also uniform in time.

To find the distribution function in a nonequilibrium process one should solve the Liouville equation allowing for the dynamics of all systems entering the ensemble. When considering the evolution of the initial equilibrium ensemble (depending on energy only) in an adiabatic process, it is convenient to write the general solution of Liouville equation in energy coordinates $(E,-t)[42]$ canonically conjugated to the coordinates $(p, x)$ in which the coordinate $E \in[0, \infty]$ (the total energy) fixes the phase trajectory on the phase plane and the coordinate $t \in[0, T(E, L)]$ fixes the position of the system on the phase trajectory. The general solution of the Liouville equation in the coordinates $(E,-t)$ has the form

$\rho(E,-t)=\int_{0}^{\infty} \int_{0}^{T\left(E_{0}, L_{0}\right)} \delta\left(E-E\left(E_{0},-t_{0}\right)\right) \delta\left(-t+t\left(E_{0},-t_{0}\right)\right) \rho_{0}\left(E_{0},-t_{0}\right) \mathrm{d} E_{0} \mathrm{~d}\left(-t_{0}\right)$

where $E\left(E_{0},-t_{0}\right)$ and $t\left(E_{0},-t_{0}\right)$ are laws of evolution of the systems entering the ensemble and $T\left(E_{0},-t_{0}\right)$ is the initial period of oscillations of the system with energy $E_{0}$.

In an adiabatic process the energy evolution law $E\left(E_{0}\right)$ (or $\left.E_{0}(E)\right)$ is determined by the constancy condition for the adiabatic invariant $G(E, L)$; the final total energy $E$ of the system depends only on one initial condition - the initial total energy $E_{0}$ and does not depend on the position of the system on the phase trajectory, i.e. on $t_{0}$. Uniformity of the distribution in time in an adiabatic process will hold provided that the wall velocity is adiabatically switched on. If this condition is not met (wall motion starts with a nonzero velocity), then uniformity in time will be violated in spite of the low velocity.

We shall henceforth assume that in an adiabatic process the initial uniformity in time is not violated. In this case, the relation between $t_{0}$ and $t$ takes on a simple form $t / t_{0}=T(E, L) / T\left(E_{0}, L_{0}\right)$. Taking into account what has been said above and passing over in expression (45) from the initial variables $\left(E_{0},-t_{0}\right)$ to the variables $(E,-t)$ we arrive at the distribution function which comes from the initial equilibrium distribution function in an adiabatic process

$$
\rho(E,-t)=\rho\left(E_{0}(E)\right) \frac{T\left(E_{0}, L_{0}\right)}{T(E, L)} \frac{\mathrm{d} E_{0}}{\mathrm{~d} E}
$$


Considering that in an adiabatic process $\mathrm{d} G(E, L) / \mathrm{d} E=T(E, L)[43]$ or $\mathrm{d} E_{0} / \mathrm{d} E=$ $T(E, L) / T\left(E_{0}, L_{0}\right)$, from expression (46) we obtain the distribution function which comes from the initial canonical function in an adiabatic process

$$
\rho_{\mathrm{is}}(E,-t)=\rho_{\mathrm{c}}\left(E_{0}(E)\right)=\frac{\exp \left(-E_{0}(E) / \Theta_{0}\right)}{\int_{0}^{\infty} T\left(E, L_{0}\right) \exp \left(-E / \Theta_{0}\right) \mathrm{d} E} .
$$

For $L=L_{0}$ we have $E_{0}(E)=E$ and expression (47) passes over to the initial canonical distribution function $\rho_{c, 0}$.

The adiabatic distribution function (47) identically meets the entropy constancy condition $S_{\text {is }}=\left\langle\ln \rho_{\text {is }}\right\rangle_{\text {is }}=$ const, where $\langle\ldots\rangle_{\text {is }}$ is averaging over the adiabatic distribution function. For quasi-equilibrium canonical distribution function this condition is not an identity, but can be used to find the quasi-equilibrium temperature $\Theta_{c}$ in an adiabatic process.

In general case, adiabatic and quasi-equilibrium canonical ensembles for small systems differ not only in fluctuations but also in average quantities (see $[44,45]$ and references therein). At the same time, in the case of a linear relation between the initial and final total energies of the system in an adiabatic process, the adiabatic ensemble coincides with the quasi-equilibrium canonical one. The heat capacity $C=$ $\partial\langle E\rangle_{\mathrm{c}} / \partial \Theta$ of the system is then conserved in the adiabatic process and is equal to its initial value $C_{0}$.

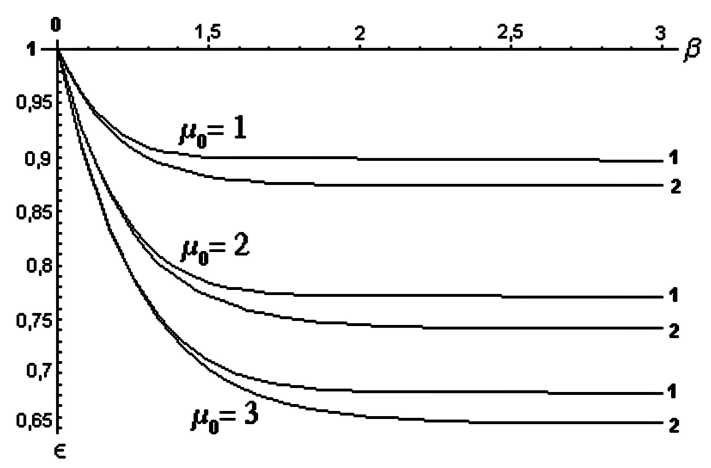

Figure 2. Adiabatic (1) and quasi-equilibrium (2) average total energies $\epsilon$ normalized to the initial value versus the extension parameter $\beta=L / L_{0}$ for different values of the initial parameter $\mu_{0}=\Theta_{0} / U\left(L_{0}\right)$ for a bounded oscillator.

For the considered systems of independent bounded oscillators and symmetric Coulomb pairs the linear relation between the initial and final total energies in an adiabatic process holds in the limit $y=E / U(L) \rightarrow \infty$, where, near the walls, both these systems behave like free particles for which the relation between the initial $E_{0}$ and final $E$ total energies in an adiabatic process has the form of direct proportionality $E_{0}(E)=\beta^{2} E$, where $\beta=L / L_{0}$. Moreover, for Coulomb pair in the limit $y \rightarrow 1$ the relation between $E_{0}$ and $E$ in an adiabatic process also becomes linear and is given by the expression

$$
E_{0}=\beta^{4 / 3} E+\frac{\alpha}{L_{0}}\left(1-\beta^{1 / 3}\right)
$$




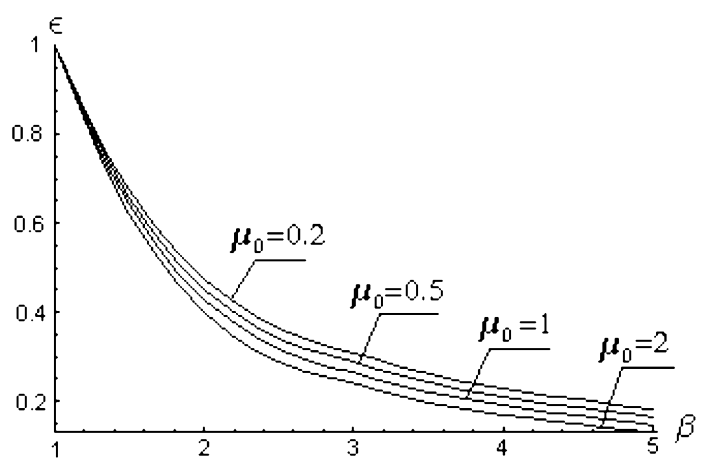

Figure 3. Adiabatic and quasi-equilibrium average total energies $\epsilon$ normalized to the initial value versus the extension parameter $\beta=L / L_{0}$ for different values of the initial parameter $\mu_{0}=\Theta_{0} / U\left(L_{0}\right)$ for a symmetric Coulomb pair.

as follows from the formula $v L^{2 / 3}=$ const. Note, for the bounded oscillator in the limit $y \rightarrow 1$, as mentioned above, the relation between the initial and final total energies in an adiabatic process vanishes at point $\beta^{\star}=L^{\star} / L_{0}$, where $E=U\left(L^{\star}\right)$. Such a distinction in the behavior of the considered system in the limit $y \rightarrow 1$ has a significant effect on the behavior of average energies in an adiabatic and quasiequilibrium canonical ensembles.

Figures 2 and 3 present $\beta$-dependence of the normalized on initial values average adiabatic and quasi-equilibrium canonical total energies for various values of the initial parameter $\mu_{0}=\Theta_{0} / U\left(L_{0}\right)$ for bounded oscillator and Coulomb pair, respectively.

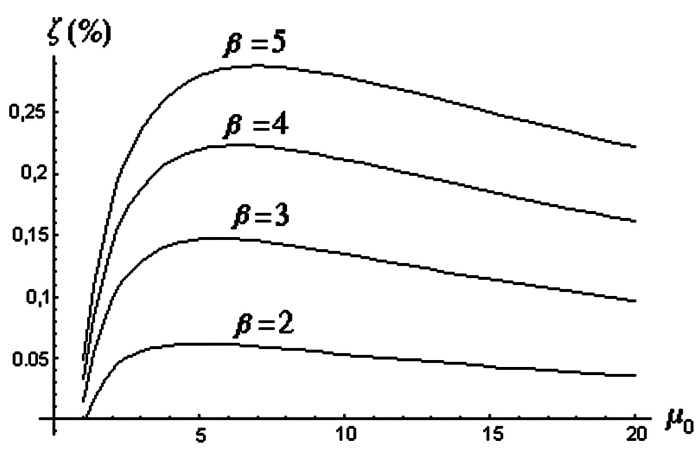

Figure 4. Quantity $\zeta=\left(\langle E\rangle_{\mathrm{s}}-\langle E\rangle_{\mathrm{c}}\right) /\langle E\rangle_{\mathrm{c}} \cdot 100 \%$ versus the initial parameter $\mu_{0}$ for various values of the extension parameter $\beta$ for a symmetric Coulomb pair.

For Coulomb pair, average adiabatic and quasi-equilibrium canonical total energies practically coincide within the given graphical accuracy, as shown in figure 3. The relative difference of average energies for Coulomb pair as a function of the initial parameter $\mu_{0}$ for different $\beta$ values is given in figure 4 . The figure shows that this difference first increases and then, on passing through the maximum, begins decreasing and that the position and value of this maximum essentially depend on 
$\beta$. This is explained by the fact that in the limits $\mu_{0} \rightarrow 0$ and $\mu_{0} \rightarrow \infty$, for most of the Coulomb pairs in the ensemble, the relation of the energies $E_{0}(E)$ in an adiabatic process has a linear form and the adiabatic distribution function tends to the canonical one. We note that for an ensemble of bounded oscillators the adiabatic distribution function has a canonical form only in the high-temperature limit $\mu_{0} \rightarrow \infty$.

In calculating the fluctuations in an adiabatic ensemble it is convinient to single out the linear part explicitly in the expressions for $E_{0}(E)$ and $E\left(E_{0}\right)$ relating the initial and the final energies in an adiabatic process:

$$
E_{0}(E)=l(\beta)+k(\beta) E+g(E, \beta),
$$

where $l$ and $k$ are coefficients independent of $E$ state and $g(E, \beta)$ is a nonlinear residue. Similarly

$$
E\left(E_{0}\right)=l_{1}(\beta)+E_{0} / k(\beta)+f\left(E_{0}, \beta\right),
$$

where the coefficient $l_{1}$ is independent of $E_{0}$ and $f\left(E_{0}, \beta\right)$ is the corresponding nonlinear residue.

The expression for the averge total energy in an adiabatic ensemble, written down in the "Schrodinger" form (the evolution is determined by the distribution function) with the use of formula (49) looks like

$$
\begin{aligned}
\langle E\rangle_{\text {is }} & =\frac{\int_{0}^{\infty} E T(E, L) \exp \left(-E_{0}(E) / \Theta_{0}\right) \mathrm{d} E}{\int_{0}^{\infty} T(E, L) \exp \left(-E_{0}(E) / \Theta_{0}\right) \mathrm{d} E}=\frac{\int_{0}^{\infty} E \mathrm{e}^{-\frac{g}{\Theta_{0}}} T(E, L) \exp (-E / \Theta) \mathrm{d} E}{\int_{0}^{\infty} \mathrm{e}^{-\frac{g}{\Theta_{0}}} T(E, L) \exp (-E / \Theta) \mathrm{d} E}= \\
& =\frac{\langle E B\rangle_{\mathrm{c}}(\Theta, L)}{\langle B\rangle_{\mathrm{c}}(\Theta, L)},
\end{aligned}
$$

where $\Theta=\Theta_{0} / k, B=\exp \left(-g / \Theta_{0}\right)$, and $\langle\ldots\rangle_{\mathrm{c}}(\Theta, L)$ is averaging over the canonical ensemble with temperature $\Theta$ and wall position $L$. Differentiation of the expression (51) with respect to $\Theta$ yields

$$
\frac{\partial\langle E\rangle_{\text {is }}}{\partial \Theta}=\frac{1}{\Theta^{2}}\left\langle(\Delta E)^{2}\right\rangle_{\text {is }},
$$

where $\left\langle(\Delta E)^{2}\right\rangle_{\text {is }}=\left\langle E^{2}\right\rangle_{\text {is }}-\left(\langle E\rangle_{\text {is }}\right)^{2}$.

Using for the $\langle E\rangle_{\text {is }}$ the "Heisenberg" representation (the evolution is determined by the total energy operator $\left.E\left(E_{0}\right)\right)$ and expression (50), we obtain

$$
\begin{aligned}
\langle E\rangle_{\text {is }} & =\frac{\int_{0}^{\infty} E\left(E_{0}\right) T\left(E_{0}, L_{0}\right) \exp \left(-E_{0} / \Theta_{0}\right) \mathrm{d} E_{0}}{\int_{0}^{\infty} T\left(E_{0}, L_{0}\right) \exp \left(-E_{0} / \Theta_{0}\right) \mathrm{d} E_{0}}=l_{1}+\frac{\langle E\rangle_{c}\left(\Theta_{0}, L_{0}\right)}{k}+\langle f\rangle_{c}\left(\Theta_{0}, L_{0}\right), \\
\frac{\partial\langle E\rangle_{\text {is }}}{\partial \Theta_{0}} & =\frac{C_{0}}{k}+\frac{\langle\Delta f \Delta E\rangle_{\mathrm{c}}\left(\Theta_{0}, L_{0}\right)}{\Theta_{0}{ }^{2}},
\end{aligned}
$$


where $C_{0}=\left\langle(\Delta E)^{2}\right\rangle_{\mathrm{c}}\left(\Theta_{0}, L_{0}\right) / \Theta_{0}{ }^{2}$ - is heat capacity of the system in the initial state and $\Delta f=f-\langle f\rangle$.

This directly implies

$$
\frac{\partial\langle E\rangle_{\text {is }}}{\partial \Theta}=C_{0}+k \frac{\langle\Delta f \Delta E\rangle_{\mathrm{c}}\left(\Theta_{0}, L_{0}\right)}{\Theta_{0}^{2}}
$$

Comparing (54) with expression (52), we finally arrive at:

$$
\left\langle(\Delta E)^{2}\right\rangle_{\text {is }}=C_{0} \Theta^{2}+\frac{1}{k(\eta)}\langle\Delta f \Delta E\rangle_{\mathrm{c}}\left(\Theta_{0}, L_{0}\right) .
$$

Fluctuations of the total energy in a quasi-equilibrium canonical ensemble are related to the heat capacity $C$ of the system as $\left\langle(\Delta E)^{2}\right\rangle_{c}=C \Theta_{\mathrm{c}}^{2}$ where $\Theta_{\mathrm{c}}$ is the temperature of the quasi-equilibrium ensemble.

In the case of a linear dependence $E_{0}(E)$ in expression (55), we have $f=\Delta f=0$, the adiabatic distribution function coincides with the quasi-equilibrium canonical function, the temperatures are $\langle 2 K\rangle_{\text {is }}=\Theta_{\mathrm{c}}=\Theta=\Theta_{0} / k(\beta)$, and the heat capacity $C$ of the system remains constant $\left(C=C_{0}\right)$ in an adiabatic process. Equation (34) will then hold identically.



Figure 5. Ratios $\xi=\left\langle(\Delta E)^{2}\right\rangle_{\text {is }} /\left\langle(\Delta E)^{2}\right\rangle_{c}$ and $C_{0} / C$ versus $\beta$ for various values of the initial parameter $\mu_{0}$ for a bounded oscillator. The family of curves (1) corresponds to the quantity $\xi$ and the family (2) corresponds to the heat capacity ratio.

If the function $E_{0}(E)$ is nonlinear, the adiabatic and quasi-equilibrium canonical ensembles differ in the average values, in particular, the adiabatic temperature $\Theta_{\text {is }}=$ $2\langle 2 K\rangle_{\text {is }}$ and the quasi-equilibrium canonical temperature $\Theta_{c}$ do not coincide with each other and with the quantity $\Theta=\Theta_{0} / k(\beta)$ in expression (55). Therefore, the relation between the adiabatic and quasi-equilibrium canonical fluctuations of the total energy will not generally be described by formula (34) which was derived for large systems in the thermodynamic limit. The difference from formula (34) is clearly seen in figure 5 which gives the ratios $\xi=\left\langle(\Delta E)^{2}\right\rangle_{\text {is }} /\left\langle(\Delta E)^{2}\right\rangle_{c}$ and $C_{0} / C$ as function of $\beta$ for different values of the initial parameter $\mu_{0}$ for the bounded oscillator. The ratio of heat capacities (the family of curves 2 ) tends to a value $1 / 2$, whereas the 


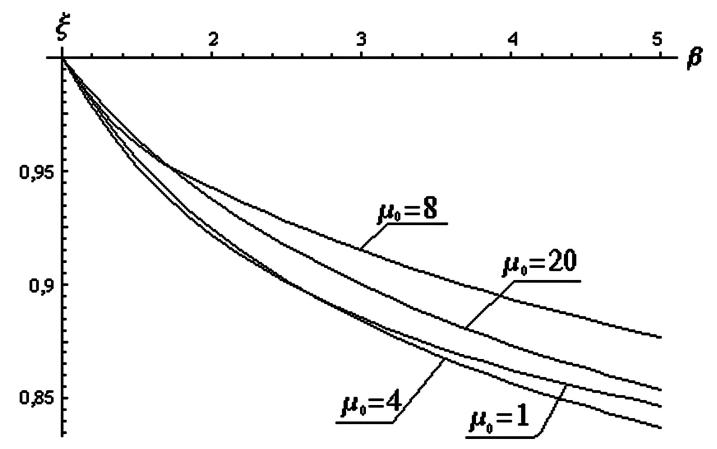

Figure 6. Dependence of the quantity $\xi=\left\langle(\Delta E)^{2}\right\rangle_{\text {is }} /\left\langle(\Delta E)^{2}\right\rangle_{c}$ on the parameter $\beta$ for various $\mu_{0}$ for a symmetric Coulomb pair.

ratio of fluctuations $\xi$ (the family of curves 1) tends to another limiting value of 0.59 .

As distinct from the bounded oscillator, for a symmetric Coulomb pair relation (34) holds to a much higher accuracy. Figure 6 presents the ratio $\xi=$ $\left\langle(\Delta E)^{2}\right\rangle_{\text {is }} /\left\langle(\Delta E)^{2}\right\rangle_{c}$ as a function of $\beta$ for various values of the initial parameter $\mu_{0}$. This ratio coincides to the graphic precision with $C_{0} / C$. The intersection of curves in the figure is due to the above-mentioned fact that the adiabatic ensemble tends to the canonical one in the limits $\mu_{0} \rightarrow 0$ and $\mu_{0} \rightarrow \infty$.

The deviation from formula (34), depicted in figure 7 , for a symmetric Coulomb pair $\lambda=\left(\left(\xi-C_{0} / C\right) / \xi\right) 100 \%$ (where $\xi$ and $C_{0} / C$ change by more than $15 \%$ ) does not exceed three percent in the considered range of parameters. Thus, the behavior of energy fluctuations in an adiabatic ensemble for Coulomb pairs turns out to be rather close to the expression that describes energy fluctuations in an adiabatic ensemble for large systems [30].

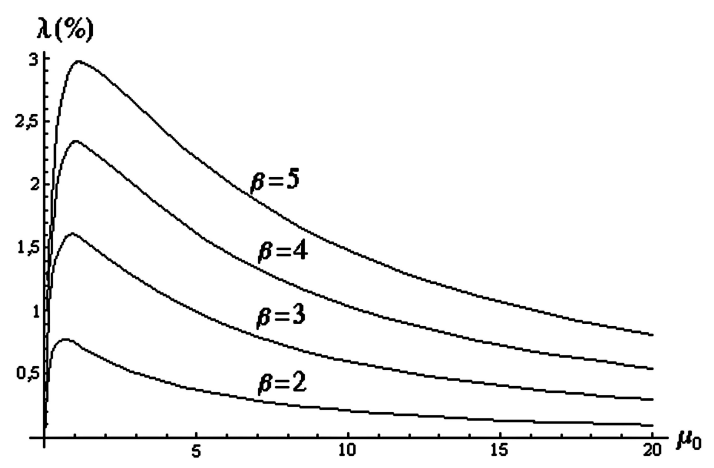

Figure 7. Dependence of $\lambda=\left(\left(\xi-C_{0} / C\right) / \xi\right) 100 \%$ on the initial parameter $\mu_{0}$ for various $\beta$ for a symmetric Coulomb pair. 


\section{Conclusion}

The results presented in this paper show that the relations of type $\chi_{s}=\chi_{\text {is }}$ play a significant role in quantum statistical mechanics of isolated systems which can attain statistical equilibrium due to internal interactions. The equality of adiabatic $\chi_{s}$ and isolated $\chi_{\text {is }}$ susceptibilities stems from the subtle form of ensemble dependence which manifests itself in the linear response theory for isolated systems. Generalizations of this equality can be effectively used in the theory of nonlinear response, in particular, to obtain an adiabatic approximation of the nonlinear response or in the description of the adiabatic ensemble which differs from the quasi-equilibrium canonical ensemble if the system heat capacity changes in the adiabatic process.

From the results obtained in this paper there also follow specific features of statistical ensembles for small (one-particle and one dimensional) nonideal independent systems - the harmonic oscillator and the symmetric Coulomb pair - whose motion is limited to motionless or slowly moving apart elastic walls. As expected, the canonical and microcanonical ensembles for such systems differ not only in their fluctuations but also in average values. Less obvious are the results of comparison of quasi-equilibrium canonical and adiabatic ensembles. These ensembles also differ in their average values (especially in the case of a bounded oscillator), but the behaviour of energy fluctuations in an adiabatic ensemble for Coulomb pairs appears to be rather close to the expression (34) describing the energy fluctuations in an adiabatic ensemble for large systems.

\section{References}

1. Landau L.D., Lifshitz E.M. Statistical Physics, 3rd ed. Oxford, Pergamon Press, 1977.

2. Klimontovitch Yu.L. Statistical Physics. Moscow, Nauka, 1982, (in Russian); Statistical Theory of Open Systems. Moscow, Yanus, 1995, (in Russian).

3. Kubo R. Statistical Mechanics. Amsterdam, North-Holland Publishing Company, 1965.

4. Wright A. // Phys. Rev., 1949, vol. 76, p. 1826.

5. Broer L.J.F. // Physica, 1951, vol. 17, p. 531.

6. Callen H.B., Welton T.A. // Phys. Rev., 1951, vol. 83, p. 34.

7. Gorelik G.S. // Uspekhi Fiz. Nauk, 1952, vol. 44, p. 33.

8. Ginzburg V.L. // Uspekhi Fiz. Nauk, 1952, vol. 46, p. 348.

9. Tatarskiy V.I. // Uspekhi Fiz. Nauk, 1987, vol. 151, p. 273.

10. Klimontovitch Yu.L. // Uspekhi Fiz. Nauk, 1987, vol. 151, p. 309.

11. Ginzburg V.L., Pitaevskiy L.P. // Uspekhi Fiz. Nauk, 1987, vol. 151, p. 333.

12. Klimontovitch Yu.L. Preprint N.8, Moscow State University, Physical Department, 2001.

13. Kubo R. // J. Phys. Soc. Japan, 1957, vol. 12, p. 570.

14. Yamamoto T. // Phys. Rev., 1960, vol. 119, p. 701.

15. Rosenfeld L. // Physica, 1961, vol. 27, p. 67.

16. Saito N. // Phys. Soc. Japan, 1961, vol. 10, p. 621.

17. Redfield A.G. // Phys. Rev., 1962, vol. 128, p. 2251. 
18. Tjon J.A. // Physica, 1964, vol. 30, p. 1314.

19. Mountain R.D. // Physica, 1964, vol. 30, p. 808.

20. Caspers W.J. Theory of Spin Relaxation. New York, Intersc. Publ, 1964.

21. Brenig W. // Z. Phys., 1967, vol. 206, p. 212.

22. Callen H., Swendsen R.H., Tahir-Kheli R. // Phys. Letters A, 1967, vol. 25, p. 505.

23. Caspers W.J. // Physica, 1968, vol. 40, p. 125.

24. Valkering T.P. // Physica, 1971, vol. 53, p. 117.

25. Caspers W.J. et al. // Physica, 1971, vol. 53, p. 210.

26. Valkering T.P., Caspers W.J. // Physica, 1973, vol. 63, p. 113.

27. Valkering T.P., Caspers W.J. // Physica, 1974, vol. 78, p. 516.

28. Samokhin A.A. // Zh. Exper. Teor. Fiz., 1966, vol. 51, p. 928.

29. Samokhin A.A. // Zh. Exper. Teor. Fiz., 1967, vol. 53, p. 360, (one of the referee of this paper was Yu.L. Klimontovich who by his own will address the author and after usefull discussion the paper had been published).

30. Samokhin A.A. // Physica, 1968, vol. 39, p. 541.

31. Samokhin A.A. // Teor. Mat. Fiz., 1970, vol. 5, p. 439.

32. Samokhin A.A. // Physics letter, 1971, vol. 36A, p. 372.

33. Samokhin A.A. // Physica, 1972, vol. 58, p. 26.

34. Mashkevich S.V., Mashkevich V.S. // Phys. Rev. E, 1995, vol. 51, p. 245.

35. Meza-Montes L., Ulloa S. // Phys. Rev. E, 1997, vol. 55, p. R6319.

36. Artuso R. et al. // Phys. Rev. E, 1997, vol. 55, p. 6384.

37. Casetti L. et al. // Phys. Rev. E, 1997, vol. 55, p. 6566.

38. Landau L.D., Lifshitz E.M. Mechanics, 3rd ed. Oxford, Pergamon Press, 1977.

39. Spitzer L. Physics of Fully Ionized Gases. New York, Interscience, 1962.

40. Artsimovich L.A., Sagdeev R.Z. Fizika Plazmy Dlya Fizikov (Plasma Physics for Physicists). Moskow, Atomizdat, 1979, (in Russian).

41. Mityugov V.V. // Uspekhi. Fiz. Nauk., 2000, vol. 170, No. 6, p. 681.

42. Bakai A.S., Stepanovskii Yu.P. Adiabaticheskie Invarianty (Adiabatic Invariants). Kiev, Naukova dumka, 1981, (in Russian).

43. Landau L.D., Lifshitz E.M. Quantum Mechanics: Non-Relativistic Theory, 3rd ed. Oxford, Pergamon Press, 1977.

44. Andreev S.N., Samokhin A.A. // Bulletin of the Lebedev Physics Institute (Kratkie Soobshcheniya po Fizike), 2000, No. 4, p. 1.

45. Andreev S.N., Samokhin A.A. // Bulletin of the Lebedev Physics Institute (Kratkie Soobshcheniya po Fizike), 2000, No. 11, p. 8. 


\title{
До статистичної механіки адіабатичного ансамблю
}

\author{
С.Н.Андрєєв, А.А.Рухадзе, А.А.Самохін
}

Інститут загальної фізики ім. О.М.Прохорова РАН Росія, 119991 Москва, вул. Вавилова, 38

Отримано 26 грудня 2003 р.

Обговорюються різноманітні підходи до опису адіабатичних процесів на основі термодинаміки та статистичної механіки. Для опису адіабатичних процесів в рамках класичної та квантової статистичної механіки використовуються рівняння для так званих адіабатичної та ізольованої сприйнятностей і їх узагальнення, а також адіабатичні інваріанти. Показано, що функція розподілу в адіабатичному ансамблі відрізняється від квазірівноважної канонічної форми при умові, що теплоємність системи при адіабатичному процесі є змінною.

Ключові слова: адіабатичні процеси, статистичні ансамблі, флуктуації, лінійний та нелінійний відгук

PACS: $05.20 . \mathrm{Gg}, 05.30 . \mathrm{Ch}$ 